\title{
Blastocladiales e Spizellomycetales do Parque Estadual da Serra da Cantareira, São Paulo, Brasil'1.
}

\author{
CRISTIANE DE ALMEIDA NASCIMENTO ${ }^{2,3}$ e CARMEN LIDIA AMORIM PIRES-ZOTTARELLI ${ }^{2}$
}

\begin{abstract}
Blastocladiales e Spizellomycetales from the Parque Estadual da Serra da Cantareira, São Paulo, São Paulo State, Brazil). To study the diversity of the Blastocladiales and Spizellomycetales, water and soil samples were collected monthly from June/2005 to June/2006, from the Parque Estadual da Serra da Cantareira, São Paulo State, Brazil. The isolation of these fungi was carried out in laboratory treating the water and soil samples by the baiting technique with cellulosic, chitinous and keratinous substrates, and using fruits submerged in water bodies as baits in the field. From 111 isolations, nine taxa were identified, three belonging to Blastocladiales and six to Spizellomycetales. Karlingia asterocysta Karling and Rozella chytriomycetis Karling are new records for Brazil.
\end{abstract}

Key words - Atlantic rainforest, diversity, fungi

RESUMO - (Blastocladiales e Spizellomycetales do Parque Estadual da Serra da Cantareira, São Paulo, Brasil). Para o estudo da diversidade de Blastocladiales e Spizellomycetales, mensalmente, de junho/2005 a junho/2006, amostras de água e solo foram coletadas no Parque Estadual da Serra da Cantareira, Estado de São Paulo. O isolamento dos fungos foi realizado no laboratório tratando as amostras de água e de solo pela técnica de iscagem com substratos celulósicos, quitinosos e queratinosos, e usando frutos submersos em corpos d'água como iscas em campo. De 111 ocorrências, nove táxons foram identificados, com três sendo pertencentes à Blastocladiales e seis a Spizellomycetales. Karlingia asterocysta Karling e Rozella chytriomycetis Karling são primeiras citações para o Brasil.

Palavras-chave - diversidade, fungos, Mata Atlântica

\section{Introdução}

Blastocladiales e Spizellomycetales são ordens inseridas no Reino Fungi, filo Blastocladiomycota e Chytridiomycota, respectivamente, sendo os únicos representantes do reino que possuem esporos móveis (flagelados). Blastocladiales engloba fungos cosmopolitas, normalmente encontrados na água e/ou no solo como sapróbios, ou como parasitas em animais microscópicos, outros fungos, larvas de mosquitos, e de muitas plantas de importância econômica. A presença de uma proeminente capa nuclear nos zoósporos e planogametas, bem como a produção de esporângios de resistência de parede espessada, usualmente punctadas, são características marcantes de seus representantes (Alexopoulos et al. 1996). A ordem é composta por cinco famílias, com 14 gêneros e 179 espécies (Kirk et al. 2008), estando atualmente inserida no filo Blastocladiomycota, o qual foi recentemente criado após a realização de estudos moleculares e ultra-estruturais, que evidenciaram que os representantes desta ordem são

1. Parte da dissertação de mestrado da primeira autora, Programa de Pós-Graduação em Biodiversidade Vegetal e Meio Ambiente do Instituto de Botânica, São Paulo, SP, Brasil.

2. Instituto de Botânica, Núcleo de Pesquisa em Micologia, Caixa Postal 3005, 01061-970 São Paulo, SP, Brasil.

3. Autor para correspondência: crisbotanic@yahoo.com.br distintos dos de Chytridiomycota (James et al. 2006, Hibbett et al. 2007).

Os organismos pertencentes à Spizellomycetales são também cosmopolitas, comumente sapróbios em substratos orgânicos, na água e no solo, e/ou parasitas. Duas famílias, sete gêneros e 27 espécies atualmente representam esta ordem (Kirk et al. 2008), as quais estão inseridas no filo Chytridiomycota, cuja conceituação foi reformulada por Hibbett et al. (2007), devido às recentes mudanças taxonômicas citadas acima. Estudos moleculares realizados por James et al. (2006) revelaram que os gêneros Rhizophlyctis, Entophlyctis, Olpidium e Rozella, incluídos em Spizellomycetales por Barr (1980), com base na ultraestrutura do zoósporo e desenvolvimento do talo, compreendem grupo polifilético. Demonstraram também que Rhizophlyctis constitui um grupo polifilético e diverso, o qual se agrupou a Catenomyces persicinus A. M. Hanson, espécie até então incluída em Blastocladiales, divergindo de Rozella e Olpidium, que passaram a compreender duas novas linhagens, totalmente separadas dos fungos verdadeiros, com Olpidium agrupando-se com Zygomycota e Rozella apresentando-se como um grupo externo divergente das demais linhagens fúngicas. No entanto, é consenso que, considerando-se a limitada quantidade de táxons incluídos nos estudos moleculares realizados até o momento, propostas de modificações taxonômicas devem ser avaliadas com muita cautela, 
uma vez que ainda há a necessidade de outros estudos que possam englobar maior número de espécies.

Diante das dimensões territoriais do Brasil, os estudos sobre fungos zoospóricos existentes até o momento ainda são escassos, sendo conduzidos por poucos especialistas e concentram-se em áreas de Mata Atlântica no Estado de São Paulo (Rogers et al. 1970, Lyra \& Milanez, 1974, Booth 1979, Milanez \& Trufem 1981, 1984, Schoenlein-Crusius et al. 1990, 1992, Schoenlein-Crusius \& Milanez 1989, 1998a, b, c, Pires-Zottarelli et al. 1996, Rocha \& Pires-Zottarelli 2002, Schoenlein-Crusius et al. 2006, Pires-Zottarelli \& Rocha 2007, Pires-Zottarelli \& Gomes 2007, Nascimento \& Pires-Zottarelli, 2009, dentre outros). A presente contribuição se refere ao levantamento desses organismos do Parque Estadual da Serra da Cantareira, importante fragmento de Mata Atlântica localizado no Estado de São Paulo, área até então inexplorada para levantamento de representantes de Blastocladiales e Spizellomycetales, ampliando assim o conhecimento da micota zoospórica no país.

\section{Material e métodos}

O Parque Estadual da Serra da Cantareira está localizado no Estado de São Paulo (2332'36" S e $46^{\circ} 37^{\prime} 59^{\prime \prime} \mathrm{W}$ ) e é considerado como a maior unidade de conservação situada dentro de um perímetro urbano. Com área de aproximadamente 7.916 ha e pela sua importância na regulação do clima, estabilização de solo, conservação de mananciais e espécies da fauna e flora ameaçadas de extinção, em 1994, foi declarado pela Unesco como parte da Reserva da Biosfera do Cinturão Verde de São Paulo (Secretaria do Meio Ambiente 2003).

Para o estudo da diversidade de Blastocladiales e Spizellomycetales, 13 coletas mensais de água e solo foram realizadas de junho de 2005 a junho de 2006 em sete pontos aleatoriamente pré-demarcados na área do Parque, totalizando 91 amostras de água e 91 de solo. As amostras foram tratadas conforme método de iscagem descrita em Milanez (1989), sendo utilizadas neste estudo as duas técnicas tradicionalmente conhecidas. Na primeira, conduzida em laboratório, substratos celulósicos (semente de Sorghum sp. cortadas ao meio, epiderme de cebola, palha de milho, grãos de pólen e celofane), quitinoso (exoesqueleto de camarão) e queratinosos (ecdise de cobra, cabelo loiro de crianças) foram adicionados às amostras de água e solo. Na segunda técnica, realizada em campo, maçãs (Malus sp.) foram acondicionadas em recipientes plásticos perfurados que permaneceram submersos nos corpos d'água por três a quatro semanas. Após este período, as maçãs foram transportadas para o laboratório e lâminas foram preparadas para observação ao microscópio das pústulas aderidas à epiderme dos frutos.
Com auxílio de literatura especializada, os espécimes foram identificados e incorporados ao herbário do Instituto de Botânica (SP), por meio de lâminas semi-permanentes preparadas com lactofenol com azul de algodão, resina polivinílica com glicerina e resina polivinílica com glicerina e lactofenol com azul de algodão (Milanez 1989, Morton et al. 1993, modificado). A classificação taxonômica adotada foi a contida no dicionário de Kirk et al. (2008).

A frequência de ocorrência das espécies, em porcentagem, foi calculada de acordo com Zak \& Willig (2004): (número de meses com ocorrência do táxon/número de meses de coleta) x 100 .

\section{Resultados e discussão}

De 182 amostras (91 de água e 91 de solo) e 111 ocorrências, três táxons de Blastocladiales e seis de Spizellomycetales foram identificados, representando os gêneros Blastocladia, Catenaria, Catenophlyctis, Karlingia e Rhizophlyctis sensu J.S. Karling, e Rozella, os quais são descritos, comentados e ilustrados, com duas espécies sendo novas citações para o Brasil. Ao longo do estudo, Catenophlyctis variabilis (Karling) Karling, Karlingia curvispinosa Karling e K. granulata Karling demonstraram a maior frequência de ocorrência (92,3\%), enquanto Blastocladia pringsheimii Reinsch, $K$. asterocysta Karling, Rhizophlyctis chitinophila Sparrow e Rozella chytriomycetis Karling foram menos frequentes $(7,7 \%)$. Alguns táxons encontrados foram exclusivos em amostras de água ou de solo, com Blastocladia pringsheimii Reinsch ocorrendo apenas na água, enquanto, Karlingia asterocysta Karling, Rhizophlyctis chitinophila Sparrow e Rozella chytriomycetis Karling em solo (tabela 1). A distribuição geográfica completa dos táxons no Brasil pode ser consultada em Milanez et al. (2007).

\section{FILO BLASTOCLADIOMYCOTA}

\section{BLASTOCLADIOMYCETES}

\section{BLASTOCLADIALES \\ BLASTOCLADIACEAE}

Blastocladia pringsheimii Reinsch, Jahrb. wiss. Bot. 11: 298. 1878.

Figura 1

Talo com célula basal cilíndrica, parte distal expandida, formando lóbulos dilatados. Zoosporângios predominantemente cilíndricos, 50-120 × 7,5-40 $\mu \mathrm{m}$, às vezes fusiformes, ocasionalmente curvos, produzidos no ápice da célula basal e, quando decíduos, deixando 
Tabela 1. Frequênca (F) dos táxons de Blastocladiales e Spizellomycetales isolados do Parque Estadual da Serra da Cantareira, de junho/2005 a junho/2006 ( $\mathrm{A}$ = água; $\mathrm{S}=$ solo).

Table 1. Frequency (F) of taxa of Blastocladiales and Spizellomycetales isolated from the Parque Estadual da Serra da Cantareira, São Paulo State, from June/2005 to June/2006 (A = water; S = soil).

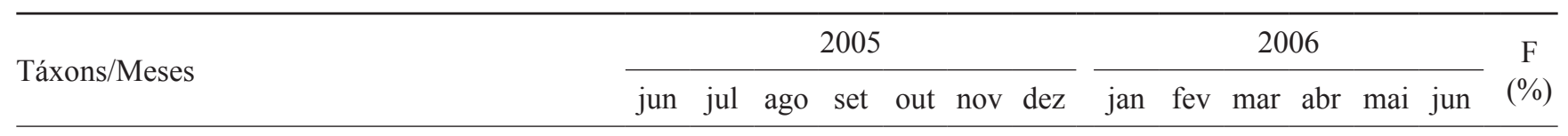

REINO FUNGI

FILO CHYTRIDIOMYCOTA

BLASTOCLADIALES

BLASTOCLADIACEAE

Blastocladia pringsheimii Reinsch

CATENARIACEAE

Catenaria sp.

Catenophlyctis variabilis (Karling) Karling

SPIZELOMYCETALES

SPIZELOMYCETACEAE

Karlingia asterocysta Karling*

K. curvispinosa Karling

K. granulata Karling

K. rosea (de Bary \& Woronin) Karling

Rhizophlyctis chitinophila (Karling) Sparrow

Rozella chytriomycii Karling*

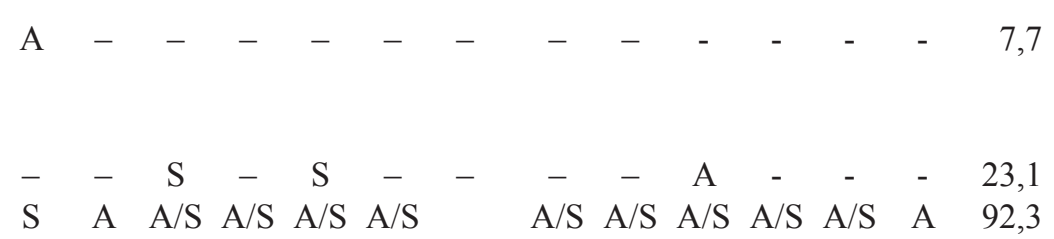

$\begin{array}{lllllllllllllllll}\mathrm{S} & - & - & - & - & - & - & - & - & - & - & - & - & 7,7\end{array}$

$\begin{array}{llllllllllllll}- & - & - & - & - & - & \mathrm{S} & \mathrm{A} & \mathrm{A} & - & \mathrm{S} & - & - & 30,8\end{array}$

$\begin{array}{llllllllllllll}\mathrm{A} & \mathrm{A} / \mathrm{S} & \mathrm{S} & \mathrm{S} & \mathrm{S} & \mathrm{S} & \mathrm{S} & \mathrm{S} & \mathrm{S} & \mathrm{S} & - & \mathrm{S} & \mathrm{S} & 92,3\end{array}$

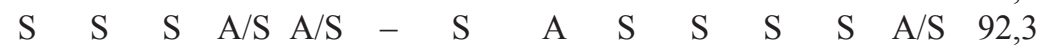

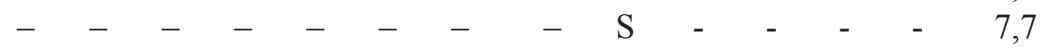

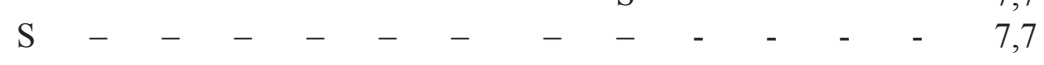

TOTAL DE ISOLAMENTOS

$\begin{array}{llllllllllllll}13 & 06 & 13 & 12 & 10 & 06 & 04 & 09 & 08 & 09 & 08 & 09 & 04 & 111\end{array}$

* Primeira referência para o Brasil / First reference to Brazil.

cicatrizes no local de inserção; sem proliferação interna; parede lisa; setas presentes. Zoósporos não observados. Esporos de resistência ovóides, 12,5-17,5 × 10-12,5 $\mu \mathrm{m}$; base truncada; parede espessada, punctada, coloração marrom; germinação não observada.

Material examinado: BRASIL. São PAULo: São Paulo, Parque Estadual da Serra da Cantareira, sapróbio em fruto de maçã (Malus sp.) submerso, 08-VI-2005, C.A. Nascimento s.n. (SP381609).

As características do espécime examinado concordam com Sparrow (1960) e Karling (1977), no entanto apresentaram zoosporângios cilíndricos e esporos de resistência normalmente menores que os descritos por Sparrow (1960) e Milanez et al. (2003), que citam, respectivamente, zoosporângios de 70-350 × 13-70 e $50-250 \times 10-60 \mu \mathrm{m}$, e esporos de 40-99 $\times 30-50$ e 36-198 $\times 28-156 \mu \mathrm{m}$. A primeira ocorrência da espécie no Brasil foi relatada por Rogers et al. (1970), de frutos submersos em corpos d'água do Parque Estadual das Fontes do Ipiranga, São Paulo, SP.

\section{CATENARIACEAE}

Catenaria $\mathrm{sp.}$

Figura 2

Taloeucárpico, policêntrico, intramatrical. Rizomicélio ramificado, extensivo. Zoosporângios inoperculados, esféricos, de 7,5-17,5 $\mu \mathrm{m}$ diâm. ou ovais, de 14-17,5 × 2-15 $\mu \mathrm{m}$, ocasionalmente irregulares, organizados em uma série linear no rizomicélio; parede lisa, coloração hialina; com um tubo de liberação, variável em tamanho. Zoósporos e esporos de resistência não observados.

Material examinado: BRASIL. São PAULo: São Paulo, Parque Estadual da Serra da Cantareira, sapróbio em ecdise de cobra, amostras de água, 31-III-2006, e amostras de solo, 11-VIII-2005, 17-XI-2005, C.A. Nascimento.

O gênero Catenaria é caracterizado pela presença de zoosporângios inoperculados organizados em série linear no rizomicélio. O táxon foi mantido em nível de gênero porque o número de gotículas lipídicas no 
zoósporo, característica taxonomicamente importante para a separação em nível específico, não foi observado nos espécimes examinados. Devido à sua rápida contaminação, os espécimes não puderam ser incorporados ao acervo da Instituição.

Catenophlyctis variabilis (Karling) Karling, Am. J. Bot. 52(2): 133-138. 1965.

Basiônimo: Phlyctorhiza variabilis Karling

Figuras 3-4

Talo eucárpico, policêntrico, intramatrical. Rizomicélio ramificado, saindo de vários pontos da parede do zoosporângio. Zoosporângios inoperculados, variáveis em tamanho e forma, geralmente irregulares, 15-62,5 × 7,5-47,5 $\mu \mathrm{m}$; ovais, $15-65 \times 12,5-52,5 \mu \mathrm{m}$; esféricos, 10-50 $\mu \mathrm{m}$ diâm., ocasionalmente clavados; organizados radialmente no rizomicélio; parede lisa, espessada, coloração hialina; com 2-3 tubos de liberação. Zoósporos e esporos de resistência não observados.

Material examinado: BRASIL. São PAULO: São Paulo, Parque Estadual da Serra da Cantareira, sapróbio em ecdise de cobra, amostras de água, 6-VII-2005, 11-VIII-2005，21-IX-2005，19-X-2005，17-XI-2005, 26-I-2006， 22-II-2006， 31-III-2006， 27-IV-2006, 19-V-2006, 5-VI-2006, e amostras de solo, 8-VI-2005, 11-VIII-2005，21-IX-2005，19-X-2005，17-XI-2005, 26-I-2006， 22-II-2006， 31-III-2006， 27-IV-2006, 19-V-2006, C.A. Nascimento s.n. (SP381610).

As características dos espécimes examinados concordam com a literatura. Neste estudo, C. variabilis apresentou crescimento sapróbio restrito ao substrato queratinoso, ecdise de cobra, concordando com Karling (1946a, 1947a), Milanez (1984a) e Pires-Zottarelli \& Rocha (2007). Karling (1965), entretanto, também cita seu crescimento em substrato celulósico. A primeira ocorrência para o Brasil é de Karling (1947a), sob a combinação Phlyctorhiza variabilis, crescendo saprofiticamente em tecidos queratinosos de insetos mortos coletados nos Estados de Roraima e Amazonas.

\section{FILO CHYTRIDIOMYCOTA}

\section{CHYTRIDIOMYCETES}

SPIZELLOMYCETALES

\section{SPIZELLOMYCETACEAE}

A princípio, espécies que apresentam vários eixos rizoidais partindo de diferentes pontos da parede do zoosporângio foram classificadas no gênero Rhizophlyctis Fischer, proposto para incluir espécies inoperculadas, e Karlingia Johanson para espécies endoperculadas (Johanson 1944, Dogma 1974). Sparrow (1960) afirmou que a endoperculação era resultado de desenvolvimento anormal em zoosporângios antigos com liberação tardia, considerando-a como caráter não constante, pouco confiável e genericamente inválido. Baseado nisto, o autor transferiu todas as espécies endoperculadas de Karlingia para o gênero Rhizophlyctis e acomodou as exoperculadas em novo gênero Karlingiomyces, por ele estabelecido. Dogma (1974) considerou Rhizophlyctis Fischer com espécies inoperculadas, Karlingiomyces Sparrow com representantes exoperculados e Karlingia Johanson como sendo um gênero endoperculado. No entanto, para Karling (1977), Karlingia é um gênero exoperculado, endoperculado ou exo-endoperculado e Rhizophlyctis abrange apenas espécies inoperculadas. Mais recentemente, Blackwell et al. (2004), em uma revisão para as espécies de Karlingiomyces, concordaram com o ponto de vista taxonômico de Sparrow (1960), exceto pela questionável inclusão de Karligiomyces granulatatus (Karling) Sparrow, Karlingiomyces lobatus (Karling) Sparrow e Karlingiomyces laevis Konno, cujos autores propõem serem exclusas deste gênero. Contudo, como os espécimes de Karlingia encontrados neste estudo apresentaram zoosporângios exoperculados, endoperculados ou exo-endoperculados, a posição de Karling (1977) foi aqui mantida, apontando os autores para a necessidade de estudos mais aprofundados com as espécies do gênero.

Chave para as espécies de Karlingia

1. Zoosporângios apresentando rizóides normalmente constritos

K. granulata

1. Zoosporângios apresentando rizóides sem constrições

K. rosea

2. Zoosporângios com conteúdo róseo

\section{2}

2. Zoosporângio com conteúdo sem coloração 3

3. Esporos de resistência âmbar, ornamentados com espinhos cônicos, numerosos, muito curvados e em forma de ganchos; funcionando como um prosporângio na germinação .... K. curvispinosa

3. Esporos de resistência marrom-esverdeados, ornamentados com espinhos cônicos pouco numerosos, geralmente retos; não funcionando como um prosporângio na germinação 

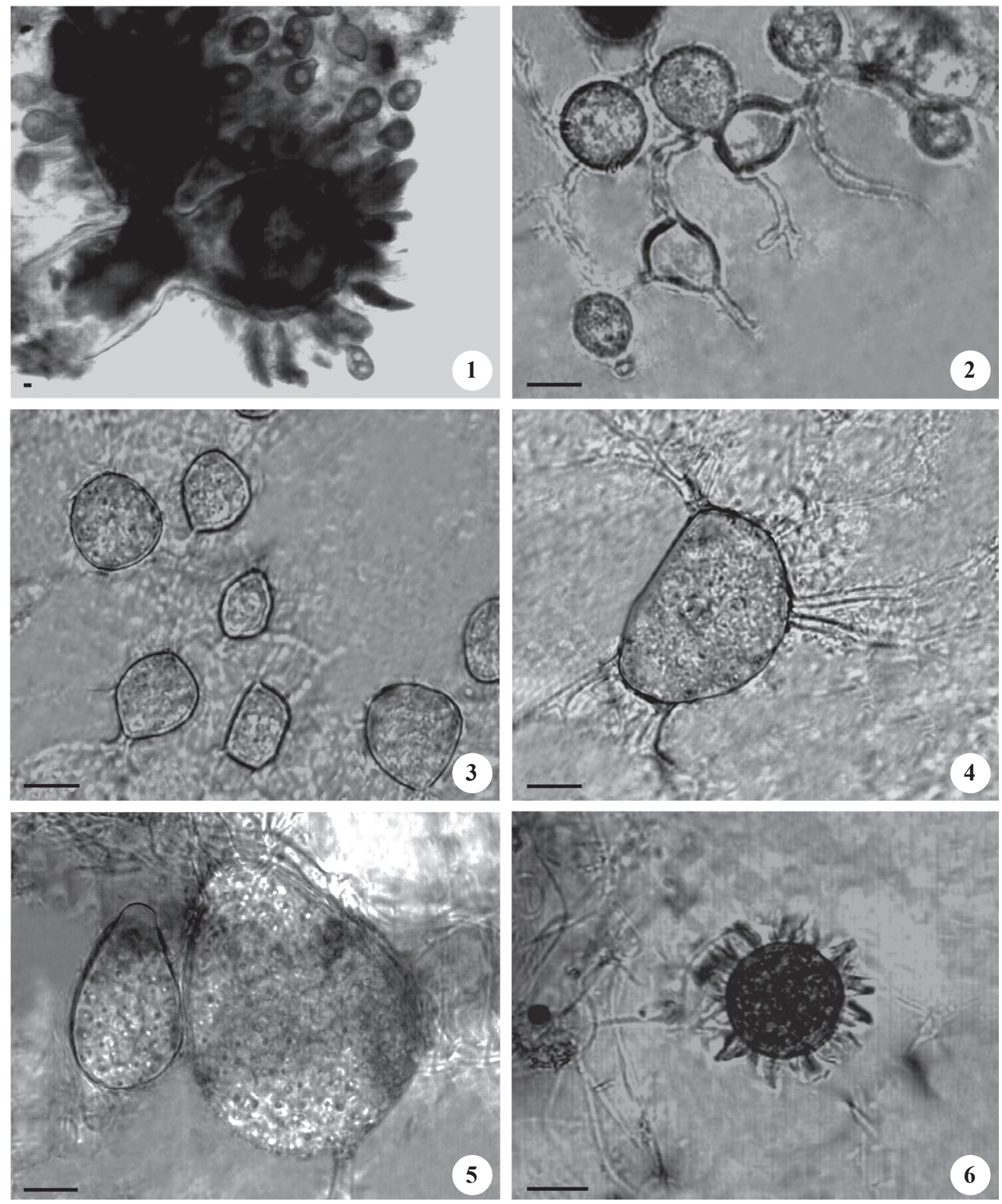

Figuras 1-6. 1. Blastocladia pringsheimii. Aspecto geral do talo evidenciando os zoosporângios e os esporos de resistência. 2. Catenaria sp. Talo policêntrico com zoosporângios em cadeia. 3-4. Catenophlyctis variabilis. 3. Talo policêntrico com zoosporângios de formas variadas. 4. Zoosporângio com vários eixos rizoidais. 5-6. Karlingia asterocysta. 5. Zoosporângios operculados. 6. Esporo de resistência ornamentado. (Barra $=10 \mu \mathrm{m})$.

Figures 1-6. 1. Blastocladia pringsheimii. Thallus with zoosporangia and resting spores. 2. Catenaria sp. Polycentric thallus with zoosporangia in chain. 3-4. Catenophlyctis variabilis. 3. Polycentric thallus with different shapes of zoosporangia. 4. Zoosporangium with multiple rhizoidal axes. 5-6. Karlingia asterocysta. 5. Operculate zoosporangia. 6. Ornamented resting spore. $(\operatorname{Bar}=10 \mu \mathrm{m})$. 
Karlingia asterocysta Karling, Mycologia, 41:509. 1949.

Figuras 5-6

Talo monocêntrico, eucárpico, extra-intramatrical. Zoosporângios exoperculados, esféricos, 42,5-97,5 $\mu \mathrm{m}$ diâm.; ovais, 22,5-65 × 30-75 $\mu \mathrm{m}$; parede lisa, coloração hialina; com cisto; $1-3$ papilas. Sistema rizoidal extensivo, ramificado, sem constrições, com vários eixos saindo de diferentes pontos da parede do zoosporângio. Zoósporos com uma gotícula lipídica hialina, liberados por meio do opérculo formado em uma papila e envolvidos por uma matriz gelatinosa, onde permanecem agrupados por um tempo antes de sua dispersão. Esporos de resistência, esféricos, 15-30 $\mu$ m diâm.; parede com ornamentações em forma de espinhos cônicos, longos ou curtos, retos, alguns levemente curvados, pouco numerosos, coloração marrom-esverdeada, conteúdo granular; germinação não observada.

Material examinado: BRASIL. São PAULo: São Paulo, Parque Estadual da Serra da Cantareira, sapróbio em exoesqueleto de camarão, amostras de solo, 8-VI-2005, C.A. Nascimento s.n. (SP381627).

As características do espécime examinado concordam com a descrição original de Karling (1949). A espécie foi isolada originalmente de amostras de água e solo, iscadas com substrato quitinoso, exoesqueleto de camarão, nos Estados Unidos. Trata-se da primeira citação para o Brasil.

Karlingia curvispinosa Karling, 41:509-511.1949. Figuras 7-9

Talo monocêntrico, eucárpico, extra-intramatrical. Zoosporângios exoperculados, ovais, 25-72,5 × 32-75 $\mu$ m; parede lisa, coloração hialina; presença de uma papila; com cisto. Sistema rizoidal extensivo, ramificado, sem constrições, com vários eixos saindo de diferentes pontos da parede do zoosporângio. Zoósporos com uma gotícula lipídica hialina, liberados por meio do opérculo formado em uma papila e envolvidos por uma matriz gelatinosa, onde permanecem agrupados por um breve período antes de sua dispersão. Esporos de resistência esféricos, 20-62,5 $\mu \mathrm{m}$ diâm., ocasionalmente ovais, $20-37,5 \times 15-27,5 \mu \mathrm{m}$; parede apresentando ornamentações em forma de espinhos cônicos, longos ou curtos, normalmente curvados, numerosos; coloração âmbar; geralmente com cisto; conteúdo finamente granular; funcionando como um prosporângio na germinação.

Material examinado: BRASIL. São PAULO: São Paulo, Parque Estadual da Serra da Cantareira, sapróbio em exoesqueleto de camarão, amostras de água,
22-II-2006, e de solo, 7-XII-2005 e 27-IV-2006, C.A. Nascimento s.n. (SP381628).

Distribuição no Brasil: São Paulo: Mogi das Cruzes (C.L.A. Pires-Zottarelli, dados não publicados).

Karling (1949) menciona zoosporângios ovais maiores que os examinados neste estudo, de 10-80 $\times$ 15-140 $\mu \mathrm{m}$ e, esporos de resistência menores, de 6-21 $\mu \mathrm{m}$ diâm. O autor observou que em alguns esporos de resistência as ornamentações foram reduzidas, dando à parede destas estruturas um aspecto verrucoso, mas tal ocorrência não foi verificada neste estudo. K. curvispinosa foi isolada pela primeira vez no Brasil de amostras de solo, iscadas com exoesqueleto de camarão, coletadas na Serra do Itapety em Mogi das Cruzes, Estado de São Paulo (C.L.A. Pires-Zottarelli, dados não publicados).

Embora se note similaridade morfológica entre os isolados de Karlingia asterocysta e K. curvispinosa, os esporos de resistência das duas espécies são bem distinguíveis pela presença de espinhos normalmente retos e espaçados nos esporos de resistências marromeverdeados de $K$. asterocysta, os quais se apresentam bastante numerosos e geralmente curvados, semelhantes a um gancho, em $K$. curvispinosa, cujo esporo de resistência apresenta coloração âmbar e funciona como um prosporângio na germinação. Outra característica muito importante não mencionada na descrição original e frequentemente observada nos espécimes examinados de $K$. curvispinosa, foi a presença de um cisto muito evidente em seus esporos de resistência, o qual não foi observado nos isolados de $K$. asterocysta.

Karlingia granulata Karling, Mycologia 39: 57-58. 1947.

Figuras 10-11

Talo monocêntrico, eucárpico, extra-intramatrical. Zoosporângios exo e/ou endoperculados, esféricos, 50-140 $\mu \mathrm{m}$ diâm., ocasionalmente ovais; parede lisa, mas tornando-se enrugada na maturidade, coloração de hialina a acinzentada; com cisto. Sistema rizoidal extensivo, ramificado, geralmente constritos. Zoósporos com várias gotículas lipídicas hialinas, liberados por meio de um opérculo formado no ápice e/ou no interior do tubo de liberação ou de uma papila e envolvidos por uma matriz gelatinosa, onde permanecem agrupados por um breve período antes de sua dispersão. Esporos de resistência não observados.

Material examinado: BRASIL. São PAulo: São Paulo, Parque Estadual da Serra da Cantareira, sapróbio em epiderme de cebola e palha de milho, amostras de água, 6-VII-2005, e de solo, 8-VI-2005, 6-VII-2005, 

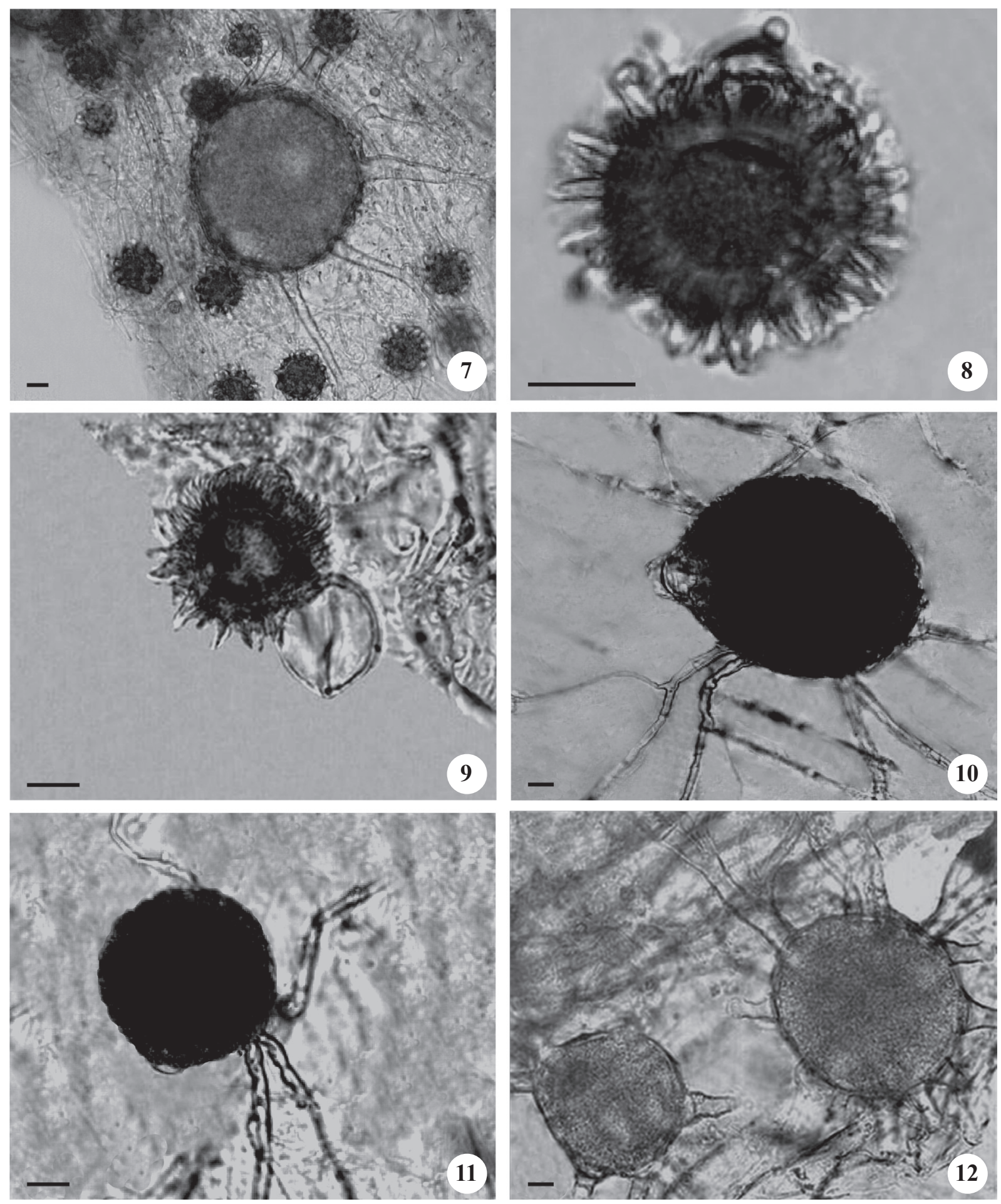

Figuras 7-12. 7-9. Karlingia curvispinosa. 7. Zoosporângio e esporos de resistência ornamentados. 8. Esporo de resistência com cisto. 9. Zoosporângio vazio originado da germinação do esporo de resistência. 10-11. Karlingia granulata. 10. Zoosporângio endoperculado com rizóides constrictos. 11. Zoosporângio exoperculado. 12. Karlingia rosea. Zoosporângios com vários tubos de liberação. (Barra $=10 \mu \mathrm{m})$.

Figures 7-12. 7-9. Karlingia curvispinosa. 7. Zoosporangium and ornamented resting spores. 8. Resting spore with cyst. 9. Empty zoosporangium originated from germination of resting spore. 10-11. Karlingia granulata. 10. Endooperculate zoosporangium with constricted rhizoids. 11. Exooperculate zoosporangium. 12. Karlingia rosea. Zoosporangia with several exit tubes. (Bar $=10 \mu \mathrm{m})$. 
11-VIII-2005，21-IX-2005，19-X-2005，17-XI-2005, 7-XII-2005,26-I-2006,22-II-2006,31-III-2006,19-V-2006 e 5-VI-2006, C.A. Nascimento s.n. (SP381625).

Os zoosporângios esféricos dos espécimes examinados se apresentaram menores que os descritos originalmente por Karling (1947b), que cita de 8-240 $\mu$ m diam. Dogma (1974) também descreve zoosporângios maiores que os observados neste estudo, de 24-180 $\mu \mathrm{m}$ diâm., no entanto, comparando com isolados brasileiros, os zoosporângios foram maiores que os descritos por Rocha \& Pires-Zottarelli (2002), que mencionam de (25-)37-50(-62) $\mu \mathrm{m}$ diâm., e Pires-Zottarelli \& Gomes (2007) que mencionam de (20-)30-55 $\mu \mathrm{m}$ diâm. Karling (1947b) comenta que esta espécie é normalmente monocêntrica, mas em raros casos pode se tornar policêntrica, característica que não foi observada nos isolados examinados. Os espécimes se desenvolveram em substratos celulósicos, concordando com Karling (1947b), Dogma (1974) e Rocha \& Pires-Zottarelli (2002). A primeira citação desta espécie no Brasil é de Karling (1947b) que a isolou de amostras de solo coletadas no Estado do Mato Grosso.

Karlingia rosea (de Bary \& Woronin) A. E. Johanson, Am. J. Bot. 31:399. 1944.

Basiônimo: Chytridium roseum de Bary \& Woronin Figura 12

Talo monocêntrico, eucárpico, extra-intramatrical. Zoosporângios endoperculados, esféricos, 35-140 $\mu \mathrm{m}$ diâm., ovais, 70-150 ×60-120 $\mu \mathrm{m}$; conteúdo róseo, tornando-se marrom-avermelhado na maturidade; parede lisa; com 1 a vários tubos de liberação, geralmente curtos. Sistema rizoidal extensivo, bastante ramificado, constrições ausentes, com vários eixos rizoidais saindo de diferentes pontos da parede do zoosporângio. Zoósporos com várias gotículas lipídicas, liberados por meio do endopérculo, e imediatamente dispersados. Esporos de resistência não observados.

Material examinado: BRASIL. São PaUlo: São Paulo, Parque Estadual da Serra da Cantareira, sapróbio em epiderme de cebola, palha de milho e celofane, amostras de água, 21-IX-2005, 19-X-2005, 26-I-2006 e 5-VI-2006, e de solo, 8-VI-2005, 6-VII-2005, 11-VIII-2005, 21-IX-2005， 19-X-2005， 17-XI-2005， 7-XII-2005, 22-II-2006，31-III-2006，27-IV-2006，19-V-2006 e 5-VI-2006, C.A. Nascimento s.n. (SP381626).

Os isolados examinados apresentaram zoosporângios esféricos menores que os descritos por Johanson (1944), que menciona de 3,3-250 $\mu \mathrm{m}$ diâm., Milanez (1984b), de 100-180 m diâm., e Rocha \& Pires-Zottarelli (2002), que citam de 53-186 $\mu \mathrm{m}$ diâm., concordando com as demais características citadas pelos autores. A primeira citação da espécie no Brasil foi feita por Joffily (1947), isolada de amostras de solo, iscadas com celofane, coletadas nos Estados da Paraíba e de São Paulo.

Rhizophlyctis chitinophila (Karling) Sparrow, Aquatic Phycomycetes 444-445. 1960.

Basiônimo: Karlingia chitinophila Karling Figuras 13-15

Talo eucárpico, monocêntrico, extra-intramatrical. Zoosporângios inoperculados, esféricos, 25-120 $\mu \mathrm{m}$ diâm., ovais, $30-135 \times 20-82,5 \mu \mathrm{m}$; parede lisa, coloração hialina; com 1-6 papilas. Sistema rizoidal extensivo, ramificado, com vários eixos saindo de diferentes pontos da parede do zoosporângio. Zoósporos com várias gotículas lipídicas, liberados por meio de um poro formado no ápice das papilas e envolvidos por uma matriz gelatinosa, onde permanecem agrupados ou imediatamente dispersados. Esporos de resistência esféricos, 7,5-22,5 $\mu \mathrm{m}$ diâm., parede lisa, espessada, coloração marrom, conteúdo granular; germinação não observada.

Material examinado: BRASIL. São PAULO: São Paulo, Parque Estadual da Serra da Cantareira, sapróbio em exoesqueleto de camarão, amostras de solo, 22-II-2006, C.A. Nascimento s.n. (SP381630).

Os zoosporângios esféricos e esporos de resistência dos espécimes examinados se apresentaram menores que os descritos por Sparrow (1960), que cita zoosporângios de 10-215 $\mu \mathrm{m}$ e esporos de resistência de 8-26 $\mu \mathrm{m}$. Rocha \& Pires-Zottarelli (2002) citam zoosporângios esféricos e ovais menores, mencionando, respectivamente, de $30-60 \mu \mathrm{m}$ e de $40-50 \times 35-45 \mu \mathrm{m}$. Os espécimes ocorreram apenas em substrato quitinoso, exoesqueleto de camarão, concordando com Sparrow (1960), Lyra \& Milanez (1974), Pires-Zottarelli \& Milanez (1993) e Rocha \& Pires-Zottarelli (2002). No Brasil, R. chitinophila foi citado por Lyra \& Milanez (1974), Pires-Zottarelli \& Milanez (1993) e Rocha \& Pires-Zottarelli (2002), mas nenhum registro da presença de endopérculo foi descrito pelos autores, concordando com Sparrow (1960), Lyra \& Milanez (1974) e Rocha \& Pires-Zottarelli (2002).

Informaçõesquantoàmorfologiaeposiçãotaxonômica desta espécie são bastante contraditórias na literatura. Existe um grupo de espécies que apresentam morfologia muito semelhante e que podem ser facilmente confundidas. Esse grupo conhecido como Rhizophlyctoid alliance inclui espécies que apresentam vários eixos rizoidais saindo de diferentes pontos da parede do zoosporângio (Dogma 

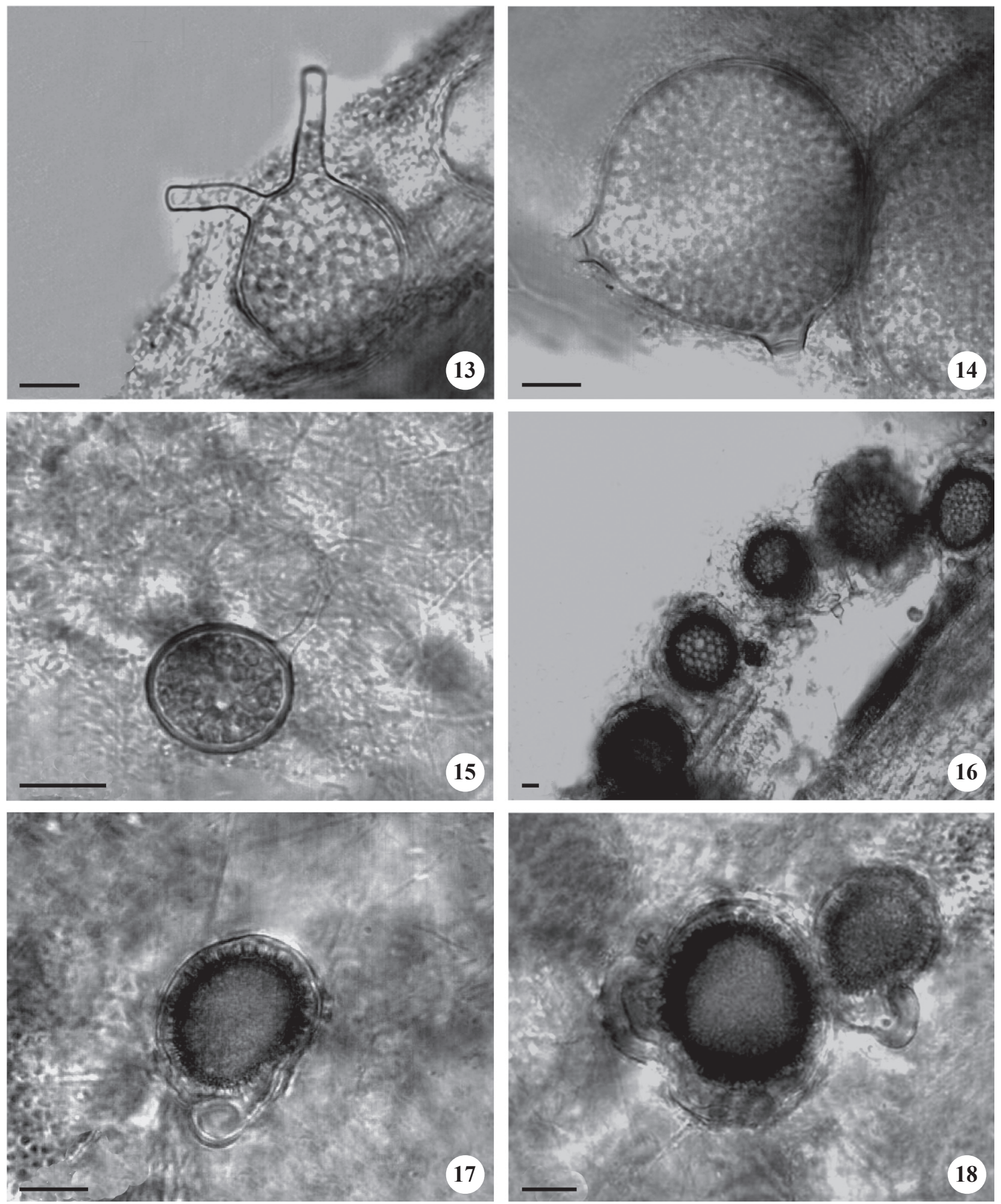

Figuras 13-18. 13-15. Rhizophlyctis chitinophila. 13. Zoosporângio com tubos de liberação longos. 14. Zoosporângio com tubos de liberação curtos. 15. Esporo de resistência. 16-18. Rozella chytriomycii. 16. Esporos de resistência. 17. Esporo de resistência em Chytriomyces appendiculatus. 18. Esporos de resistência. (Barra $=10 \mu \mathrm{m})$.

Figures 13-18. 13-15. Rhizophlyctis chitinophila. 13. Zoosporangium with long exit tubes. 14. Zoosporangium with short exit tubes. 15. Resting spore. 16-18. Rozella chytriomycii. 16. Resting spores. 17. Resting spore in Chytriomyces appendiculatus. 18. Resting spores. $(\mathrm{Bar}=10 \mu \mathrm{m})$. 
1974). Essa aliança é dividida por Sparrow (1960) em Rhizophlyctis Fischer e Karlingiomyces Sparrow, por Dogma (1974) em Rhizophlyctis Fischer, Karlingiomyces Saprrow e Karlingia Johanson, e por Karling (1977) em Rhizophlyctis Fischer e Karlingia Johanson, todos eles distinguíveis pela presença ou ausência de endopérculo e exopérculo, e pela posição que é formado. Rhizophlyctis sensu Sparrow é inoperculado ou endoperculado e Karlingiomyces é exo e/ou endoperculado, com rizóides constritos. Karlingia sensu Karling é exoperculada, endoperculada ou exo-endoperculada e Rhizophlyctis abrange apenas espécies inoperculadas. Rhizophlyctis sensu Dogma é inoperculado, Karlingia é endoperculado e Karlingiomyces é exoperculado e apresenta rizóides constritos. Neste estudo, como consta na descrição acima, observações minuciosas dos espécimes isolados não revelaram a presença de opérculo no zoosporângio e o material foi classificado em Rhizophlyctis sensu Karling. O primeiro relato da espécie no Brasil foi feito por Lyra \& Milanez (1974), isolada de amostras de água, iscadas com exoesqueleto de camarão, coletadas em São Paulo, SP.

\section{Incertae sedis}

Rozella chytriomycetis Karling, Mycologia 38:107. 1946 Figuras 16-18

Talo monocêntrico, holocárpico, endobiótico. Zoosporângios não observados. Esporos de resistência esféricos, 12,5-40 $\mu \mathrm{m}$ diâm., ovais, 22,5-32,5 × 17,5$27,5 \mu \mathrm{m}$; conteúdo granular, com um grande vacúolo central; parede com ornamentações em forma de espinhos equinulados, numerosos e curtos, espessada, com coloração marrom; germinação não observada.

Material examinado: BRASIL. SÃo PAUlo: São Paulo, Parque Estadual da Serra da Cantareira, parasita em Chytriomyces sp., amostras de solo, 8-VI-2005, C.A. Nascimento s.n. (SP381631).

Os espécimes examinados apresentaram esporos de resistência esféricos maiores que os descritos por Karling (1946b), que cita de 7-20 ㅆm diâm, concordando com as demais características citadas pelo autor. $\mathrm{O}$ autor descreveu a espécie parasitando Chytriomyces hyalinus Karling, de amostras de água, coletadas nos Estados Unidos, apesar de várias tentativas, sem sucesso, de inoculação em outras espécies de quitrídias. Esta é a primeira citação da espécie para o Brasil.

Os resultados encontrados contribuem significativamente para o conhecimento de Blastocladiales e Spizellomycetales, complementando um estudo de Chytridiomycota anteriormente realizado no local por Nascimento \& Pires-Zottarelli (2007). Sendo este um levantamento pontual, quando considerada toda a área que o Parque compreende, destaca-se a necessidade da conservação do local para a realização de futuros estudos.

Agradecimentos - Ao Conselho Nacional de Pesquisa e Desenvolvimento (CNPq), pela concessão de bolsa de mestrado à primeira autora e de Produtividade em Pesquisa à segunda autora; ao Instituto de Botânica de São Paulo pela infra-estrutura oferecida para o desenvolvimento do trabalho e ao Instituto Florestal pela autorização concedida para a realização das coletas.

\section{Referências bibliográficas}

ALEXOPOULOS, C.J., MIMS, C.W. \& BLACKWELL, M. 1996. Introductory Mycology. $4^{\text {th }}$ ed., John Wiley, Sons, Inc., New York.

BLACKWELL, W.H., LETCHER, P.M. \& POWELL, M.J. 2004. Synopsis and systematic reconsideration of Karlingiomyces (Chytridiomycota). Mycotaxon 89:259276.

BARR, D.J.S. 1980. An outline for the reclassification of the Chytridiales, and for a new order, the Spizellomycetales. Canadian Journal of Botany 58:2380-2394.

BOOTH, T. 1979. Strategies for study of fungi in marine and marine influenced ecosystems. Revista de Microbiologia 10:123-138.

DOGMA JR., I.J. 1974. Developmental and taxonomic studies of rhizophlyctoid fungi, Chytridiales. IV. Karlingia granulata, Karlingia spinosa, and Karlingiomyces dubius. Nova Hedwigia 25:91-105.

HIBBET, D.S., BINDER, M., BISCHOFF, J.F. et al. 2007. A higher-level phylogenetic classification of the fungi. Mycological Research 111:509-547.

JAMES, T.Y.,LETCHER,P.M.,LONGCORE, J.E., MOZLEYSTANDIRDGE, S.E., PORTER, D., POWELL, M.J., GRIFFITH, G.W. \& VILGAYS, R. 2006. A molecular phylogeny of the flagellated fungi (Chytridiomycota) description of a new phylum (Blastocladiomycota). Mycologia 98:860-871.

JOHANSON, A.E. 1944. An endo-operculate chytridiaceous fungus: Karlingia rosea gen. nov. American Journal of Botany 31:397-404.

JOFFILY, I.M. 1947. Alguns ficomicetos aquáticos e terrícolas do Brasil. Boletim da Sociedade Brasileira de Agronomia 10:95-113.

KARLING, J.S. 1946a. Keratinophilic chytrids. American Journal of Botany 33:219.

KARLING, J.S. 1946b. Two new chytrid parasites of Chytriomyces. Mycologia 38:103-109.

KARLING, J.S. 1947a. Keratinophilic chytrids. II. Phlyctorhiza variabilis n. sp. American Journal of Botany 34:27-32 
KARLING, J.S. 1947b. Brazilian chytrids. X. New species with sunken opercula. Mycologia 39:56-70.

KARLING, J.S. 1949. New monocentric eucarpic operculate chytrids from Maryland. Mycologia 41:505-522.

KARLING, J.S. 1965. Some zoosporic fungi of New Zealand. I. Sydowia 19:213-226.

KARLING, J.S. 1977. Chytridiomycetarum iconographia. Vaduz: J. Cramer, New York.

KIRK, P.M., CANNON, P.F., MINTER, D.W. \& STALPERS, J.A. 2008. Dictionary of fungi. $10^{\text {th }}$ ed., CABI Bioscience, Wallingford.

LYRA, N.P. \& MILANEZ, A.I. 1974. Notas para o levantamento dos ficomicetos aquáticos do estado de São Paulo. Instituto de Micologia da Universidade Federal de Pernambuco, v.698, p.1-27.

MILANEZ, A.I. 1984a. Fungos zoospóricos do estado de São Paulo. II. Chytridiomycetes da Região Oeste. Rickia 11:115-127.

MILANEZ, A.I. 1984b. Distribuição geográfica de Karlingia rosea (De Bary \& Woronin) Johanson no Brasil. In Anais do IV Congresso da Sociedade Botânica de São Paulo. Rio de Janeiro, p.73-76.

MILANEZ, A.I. 1989. Fungos de águas continentais. In Técnicas de coleta, preservação e herborização de material botânico (O. Fidalgo \& V.L. Bononi, coords.). Série Documentos. São Paulo, Instituto de Botânica, p.17-20.

MILANEZ, A.I. \& TRUFEM, S.F.B. 1981. Ficomicetos em frutos submersos do Parque Estadual das Fontes do Ipiranga. Rickia 9:7-15.

MILANEZ, A.I. \& TRUFEM, S.F.B. 1984. Fungos zoospóricos em frutos submersos no Parque Estadual das Fontes do Ipiranga. Rickia 11:77-84.

MILANEZ, A.I., PIRES-ZOTTARELLI, C.L.A., SCHOENLEIN-CRUSIUS, I.H. \& LOHMANN, L.G. 2003. Criptógamos do Parque Estadual das Fontes do Ipiranga, São Paulo, SP. Fungos, 8: Blatocladiales. Hoehnea 30:21-29.

MILANEZ, A.I., PIRES-ZOTTARELLI, C.L.A. \& GOMES, A.L. (eds.). 2007. Brazilian zoosporic Fungi. CNPq, São Paulo.

MORTON, J.B., BENTIVENGA, S.P. \& WHEELER, W.W. 1993. Germ plasm in the International Collection of Arbuscular and Vesicular-arbuscular Mycorrhizal Fungi (INVAM) and procedures for culture development, documentation and storage. Mycotaxon 48:491-528.

NASCIMENTO, C.A. \& PIRES-ZOTTARELLI, C.L.A. 2009. Chytridiales (Chytridiomycota) do Parque Estadual da Serra da Cantareira. Acta Botanica Brasilica 23:459-473.

PIRES-ZOTTARELLI, C.L.A. \& MILANEZ, A.I. 1993. Fungos zoospóricos da Represa do Lobo ("Broa"). Novas citações para o Brasil. Revista Brasileira de Botânica 16:205-220.
PIRES-ZOTTARELLI, C.L.A. \& GOMES, A.L. 2007. Contribuição para o conhecimento de Chytridiomycota da "Reserva Biológica de Paranapiacaba", Santo André, SP, Brasil. Biota Neotropica 3:309-329.

PIRES-ZOTTARELLI, C.L.A. \& ROCHA, M. 2007. Novas citações de Chytridiomycota e Oomycota para o Parque Estadual das Fontes do Ipiranga (PEFI), SP, Brasil. Acta Botanica Brasilica 21:125-136.

PIRES-ZOTTARELLI, C.L.A., MILANEZ, A.I., SCHOENLEIN-CRUSIUS, I.H. \& LOHMANN, L.G. 1996. Criptógamos do Parque Estadual das Fontes do Ipiranga, São Paulo, SP. Fungos, 6: Chytridiales. Hoehnea 23:77-90.

ROCHA, M. \& PIRES-ZOTTARELLI, C.L.A. 2002. Chytridiomycota e Oomycota da Represa de Guarapiranga, São Paulo, SP. Acta Botanica Brasilica 16:287-309.

ROGERS, A.L., MILANEZ, A.I. \& BENEKE, E.S. 1970. Additional aquatic fungi from São Paulo State. Rickia 5:93-110.

SCHOENLEIN-CRUSIUS, I.H. \& MILANEZ, A.I. 1989. Sucessão fúngica em folhas de Ficus microcarpa L.f. submersas no lago frontal situado no Parque Estadual das Fontes do Ipiranga, São Paulo, SP. Revista de Microbiologia 20:95-101.

SCHOENLEIN-CRUSIUS, I.H. \& MILANEZ, A.I. 1998a. Fungos zoospóricos (Mastigomycotina) da Mata Atlântica da Reserva Biológica do Alto da Serra de Paranapiacaba, município de Santo André, SP. Revista Brasileira de Botânica 2:177-181.

SCHOENLEIN-CRUSIUS, I.H. \& MILANEZ, A.I. 1998b. Fungos microscópicos da Mata Atlântica de Paranapiacaba, São Paulo, Brasil. Revista Brasileira de Botânica 21:73-79.

SCHOENLEIN-CRUSIUS, I.H. \& MILANEZ, A.I. 1998c. Fungi in stream in the atlântic Rainforest in the "Reserva Biológica de Paranapiacaba", São Paulo State, Brazil. In Anais do IV Simpósio de Ecossitemas Brasileiros. ACIESP, Águas de Lindóia, p.388-394.

SCHOENLEIN-CRUSIUS, I.H., PIRES-ZOTTARELLI, C.L.A. \& MILANEZ, A.I. 1990. Sucessão fúngica em folhas de Quercus robur L. (carvalho) submersas em um lago situado no município de Itapecerica da Serra, SP. Revista de Microbiologia 21:61-67.

SCHOENLEIN-CRUSIUS, I.H., PIRES-ZOTTARELLI, C.L.A. \& MILANEZ, A.I. 1992. Aquatic fungi in leaves submerged in a stream in the Atlantic rainforest. Revista de Microbiologia 23:167-171.

SCHOENLEIN-CRUSIUS, I.H., MILANEZ, A.I., TRUFEM, S.B., PIRES-ZOTTARELLI, C.L.A, GRANDI, R.P., SANTOS, M.L. \& GIUSTRA, K.C. 2006. Microscopic fungi the Atlantic Rainsforest in Cubatão, São Paulo, Brazil. Brazilian Journal of Microbiology 37:244-252. 
SECRETARIA DO MEIO AMBIENTE. 2003. Roteiro de Visitação do Parque Estadual da Cantareira. São Paulo. SPARROW JR., F.K. 1960. Aquatic Phycomycetes. University of Michigan Press, Ann Arbor, Michigan.
ZAK, J.C. \& WILLIG, M.R. 2004. Fungal biodiversity patterns In Biodiversity of fungi. (G.M. Müeller, G.F. Bills \& M.S. Foster, eds.). Elsevier Academic Press, San Diego, p.59-75. 